\title{
JATOS DE BAIXOS NÍVEIS NO SUDOESTE DA AMAZÔNIA
}

\author{
${ }^{1}$ Raoni Santana, ${ }^{2}$ Júlio Tota, ${ }^{3}$ Rosa Maria dos Santos, ${ }^{1}$ Roseilson do Vale
}

\author{
${ }^{1}$ Doutorando em Clima e Ambiente - INPA/UEA \\ ${ }^{2}$ Universidade Federal do Oeste do Pará - UFOPA \\ ${ }^{3}$ Universidade do Estado do Amazonas - UEA
}

\section{RESUMO}

Este estudo apresenta uma análise da ocorrência de Jatos de Baixos Níveis (JBNs) em uma área de pastagem no sudoeste da Amazônia. Utilizando dados do perfil vertical de velocidade do vento, obtidos por meio de radiossondagem atmosférica, observou-se que em cerca de $60 \%$ do total de perfis analisados, apresentaram JBNs. Este percentual é bem superior ao encontrado em vários trabalhos realizados na Amazônia e está ligado ao critério de seleção de JBNs utilizado neste trabalho. Outro resultado interessante é a tendência de JBNs fracos estarem associados a alturas menores, ao passo que, JBNs fortes preferencialmente se desenvolvem em maiores alturas. Este resultado sugere que diferentes mecanismos podem ter gerado os JBNs de diferentes intensidades.

\begin{abstract}
This study presents an analysis of the occurrence of Low Level Jets (LLJs) in a grassland area in the southwestern Amazon. Using the wind vertical profile data obtained by atmospheric radiosonde, it was observed that about $60 \%$ of total analyzed profiles showed LLJs. This percentage is higher than found in several studies in the Amazon and is linked to the LLJs selection criteria used in this work. Another interesting result is the tendency of weak LLJs is associated with lower heights, whereas strong LLJs preferentially develop in greater heights. This result suggests that different mechanisms may have generated the LLJs of different intensities.
\end{abstract}




\section{INTRODUÇÃO}

Em micrometerologia, os Jatos de Baixos Níveis (JBNs) podem definidos como máximos que ocorrem no perfil vertical da velocidade do vento nos primeiros metros da atmosfera, dentro ou próximo da Camada Limite Atmosférica (CLA). Na Amazônia os JBNs vêm sendo documentados desde 1992 (Greco et. al., 1992) e, desde então, vários estudos demonstraram que não é rara a sua presença na fase noturna da CLA dessa região (Santos, 2005; Nogueira, 2008). No entanto, o percentual de JBNs encontrados nestes estudos é bem menor do aquele encontrado em regiões extratropicais em trabalhos mais recentes (Karipot et. al., 2008; 2009; Duarte et. al; 20012). Este fato pode ser explicado por duas situações: as regiões extratropicais estão mais susceptíveis a mecanismos que podem acelerar o vento na CLA, tais como, baroclinicidade de escala sinótica e efeito de Blackadar (Blackadar, 1957; Stull, 1988); os critérios de classificação de JBNs aplicados em estudos realizados na Amazônia podem não ser os mais adequados para capturar todos os perfis de vento que apresentam características de JBNs.

Baseado na segunda hipótese, este estudo apresenta uma análise da ocorrência dos JBNs no sudoeste da Amazônia, propondo critérios de classificação destes jatos, fundamentado em uma análise prévia ao conjunto de dados, que busca contemplar o maior número possível de casos de JBNs, da forma como foi posta por Banta et. al. (2002). Além disso, os casos de JBNs encontrados foram classificados de acordo com sua intensidade, para tentar inferir os possíveis mecanismos que podem ter acelerado o vento, provocando JBNs.

\section{MATERIAL E MÉTODO}

Os dados utilizados foram coletados durante a Campanha de Mesoescala Atmosférica da Estação Úmida ("Wet Season Atmospheric Mesoscale Campaign") do experimento LBA WetAMC-LBA, realizada nos meses de janeiro e fevereiro de 1999. Nesse estudo foram utilizados, basicamente, perfis verticais da velocidade do vento, coletados por radiossondagens atmosféricas. Para obter tais dados, foram lançadas 8 radiossondas em intervalos de 3 horas (no período intensivo) e seis delas em intervalos de 4 horas (no período não intensivo), cobrindo 24 horas. Aqui apenas perfis de 17 hora local (HL) as $08 \mathrm{HL}$ foram utilizados, totalizando um máximo de 5 perfis por noite. 
Para selecionar JBNs uma adaptação do método proposto por Andreas et al. (2000) foi utilizado. Considerou-se JBNs, casos em que o núcleo do jato esteve abaixo de $1000 \mathrm{~m}$ e a velocidade do vento neste ponto foi maior ou igual a $4 \mathrm{~ms}^{-1}$ e no mínimo $1 \mathrm{~ms}^{-1}$ acima e abaixo do núcleo. As razões para a escolha desses critérios serão discutidas mais adiante. Depois de identificados, os JBNs foram classificados de acordo com Karipot et al. (2008), da seguinte forma: Fracos $\left(4 \mathrm{~ms}^{-1} \geq V_{j}<7 \mathrm{~ms}^{-1}\right)$; Intermediários $\quad\left(7 \mathrm{~ms}^{-1} \geq V_{j}<\right.$ $\left.10 \mathrm{~ms}^{-1}\right)$; e Fortes $\left(V_{j}>10 \mathrm{~ms}^{-1}\right)$.

\section{RESULTADOS}

Os JBNs foram presença constante nas noites estudadas. Cerca de $60 \%$ dos perfis de vento analisados apresentaram tais características (figura 1 a), ou seja, 142 perfis de um total de 235 perfis foram considerados como JBNs, sendo destes 76 classificados como casos de JBNs fracos (53\%), 45 como JBNs intermediários (32\%) e 21 com JBNs fortes (15\%). Quanto à forma dos JBNs, diversas cofigurações foram observadas. Desde JBNs com uma camada de aceleração relativamente rasa (figura 1 a e d), até casos em que toda a CLA (incluindo Camada Limite Estável e Residual) esteve acelerada (figura 1 e e f). Outro fato interessante é a presença de mais de um máximo em muitos dos perfis analisados, mostrando a natureza estratificada do escoamento (figura $1 \mathbf{c}$ e e).

Apesar de JBNs de diferentes intensidades poderem ocorrer, a priori, em qualquer nível, houve uma tendência de JBNs fracos ocorrerem em alturas menores, ao passo que, JBNs fortes preferencialmente se desenvolvem em maiores alturas. Este fato pode ser evidenciado quando se separa os JBNs em classes (figura $1 \mathbf{h}$ ). Notou-se que o percentual de casos de JBNs fortes foi igual a zero abaixo dos $300 \mathrm{~m}$ de altura e que, tanto JBNs fracos, quantos intermediários, aconteceram preferencialmente abaixo de $600 \mathrm{~m}$ de altura. 

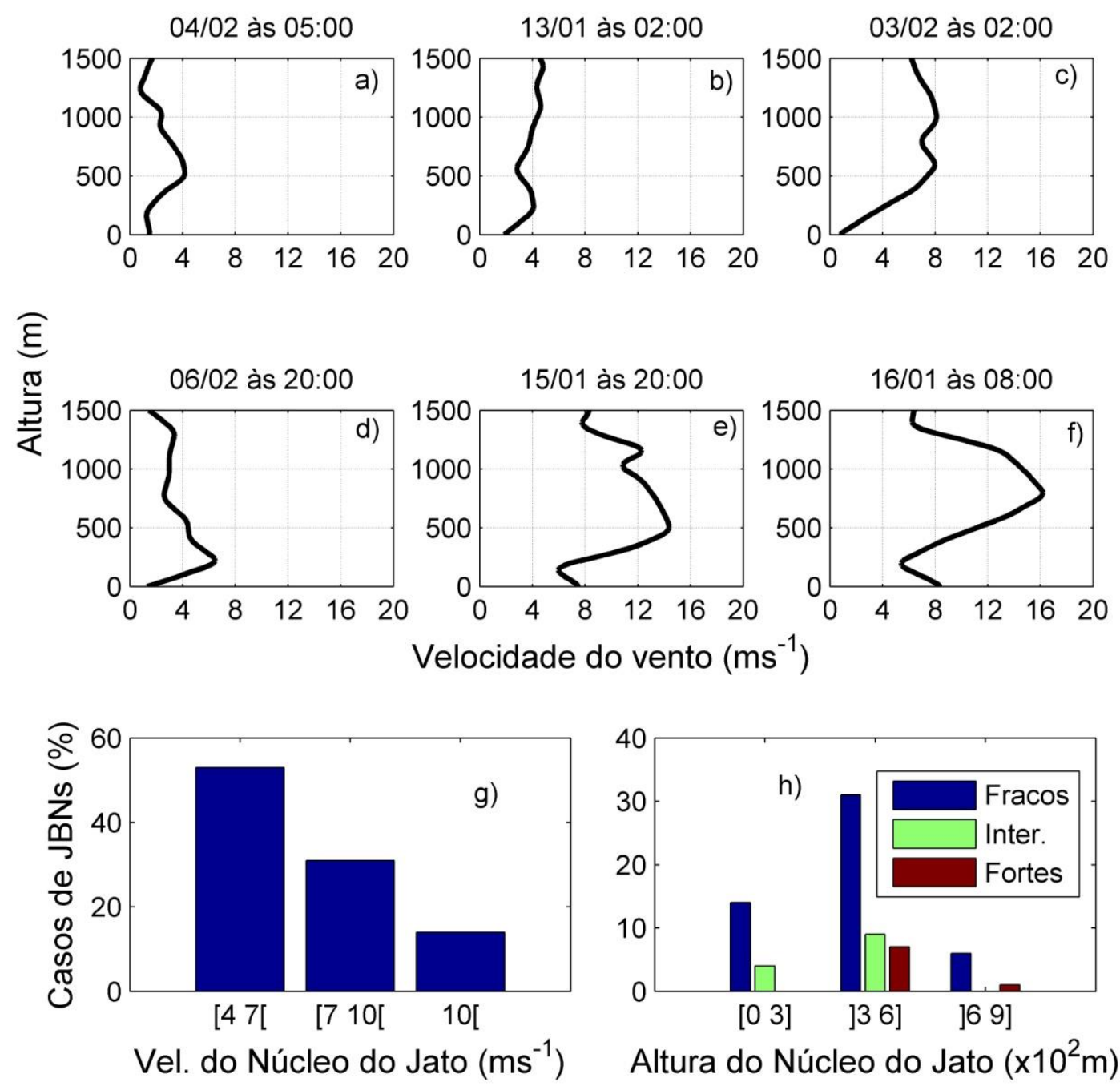

Figura 1. Exemplos de Perfis verticais de velocidade do vento que apresentaram JBNs, de acordo com os critérios de classificação adotados (a, b, c, d, e e f). Os gráficos os g e h apresentam a estatística da ocorrência dos JBNs, quanto a sua velocidade e altura, respectivamente.

\section{DISCUSSÃO}

Santos (2005), estudando JNBs em três áreas com diferentes coberturas vegetais na Amazônia (incluindo a área de estudo deste trabalho) encontrou um percentual de $20 \%$ de casos de JBNs nos perfis de vento analisados, tanto na estação seca, quanto na chuvosa. Entretanto, como a própria autora levanta, muitos dos perfis analisados apresentavam características de JBNs, mas que não foram considerados como tal, por não se adequarem ao 
critério de seleção. Este problema vai de encontro ao exposto por Banta et al. (2000), que propõe uma inspeção visual nos dados, para que o critério de seleção seja o melhor possível e englobe a maior parte dos JBNs. Neste trabalho, os $60 \%$ de JBNs encontrados, muito maior do que o encontrado por Santos (2005) e em outros trabalhos realizados na Amazônia, são explicados pelos critérios de seleção utilizados. Este procedimento tornou possível que os perfis de vento da figura $1 \mathrm{a}, \mathrm{b}$ e c, por exemplo, fossem classificados como JBNs, que apesar de apresentarem um máximo bem pronunciado, claramente são resultados de camadas mais aceleradas e podem ser importantes no processo de geração de mistura entre a altura do núcleo de jato e a superfície.

\section{CONCLUSÕES}

Neste trabalho, observou-se que em cerca de $60 \%$ do total de perfis analisados, apresentaram JBNs. Este percentual é bem superior ao encontrado em vários trabalhos realizados na Amazônia e está ligado ao critério de seleção de JBNs utilizado. Quanto à intensidade dos JBNs, existe uma tendência de JBNs fracos ocorrerem a alturas menores, ao passo que, JBNs fortes preferencialmente se desenvolvem em maiores alturas. Sugerindo que diferentes mecanismos podem ter gerado os JBNs de diferentes intensidades. Os autores acreditam que o papel desses JBNs seja de fundamental importância nos processos de trocas de energia e massa na Camada Limite Noturna da região Amazônica e que precisam ser mais bem compreendidos, por isso mais estudos devem ser realizados, utilizando equipamentos capazes de fornecer perfis de velocidade do vento com melhor resolução temporal.

\section{AGRADECIMENTOS}

Esta Pesquisa foi financiada por meio de bolsa de mestrado concedida pelo CNPq, processo n 133428/2011-7, através do programa de Pós-graduação em Clima e Ambiente INPA/UEA. Os autores agradecem ao Programa LBA pelos dados concedidos e a UEA pelo apoio logístico. 


\section{BIBLIOGRÁFICA CITADA}

Andreas, E. L.; Claffey, K. J.; Makshtas, A. 2000. Low-level atmospheric jets and inversions over the western Weddell Sea. Boundary-Layer Meteorology, 97: 459-486.

Banta, R. M.; Newsom, R. K.; Lundquist, J. K.; Pichugina, Y. L.; Coulter, R. L.; Mahrt, L. 2002. Nocturnal low-level jet characteristics over Kansas during CASES-99. BoundaryLayer Meteorology, 105: 221-252.

Blackadar, A. K. 1957. Boundary Layer Wind Maxima and their Significance for the Growth of Nocturnal Inversions. Bulletin American Meteorological Society, 38: 283-290.

Karipot, A.; Leclerc, M. Y.; Zhang, G. 2009. Characteristics of Nocturnal Low-Level Jets Observed in the North Florida Area. American Meteorological Society, 137: 2605-2621.

Karipot, A.; Leclerc, M. Y.; Zhang, G.; Keith, F. L.; Nagy, J. J. H.; Hendrey, G. R. .; Starr, G. 2008. Influence of nocturnal low-level jet on turbulence structure e $\mathrm{CO}_{2}$ flux measurements over a forest canopy. Journal of geophysical research, v113: 1-12.

Nogueira, D, S. 2008. Aspectos observacionais e numéricos da interação floresta-atmosfera na Amazônia Oriental: fenômenos turbulentos noturnos, dissertação de mestrado, Universidade Federal do Pará/Museu Paraense Emílio Goeldi/Embrapa Amazônia Oriental, Belém, Pará. 141p.

Santos, R. M. N. 2005. Estudos da camada limite noturna na Amazônia. Tese de Doutorado, Instituto Nacional de Pesquisas Espaciais, São José dos Campos, São Paulo. 177 p.

Stull, R. B. 1988. An introduction to boundary layer meteorology. Dordrecht: Kluwer Academic Publishers, 1988, 666p. 